\title{
The extremely young open cluster NGC 6611: Compiled catalogue, absorption map and the HR diagram ${ }^{\star}$
}

\author{
A.N. Belikov ${ }^{1}$, N.V. Kharchenko ${ }^{2}$, A.E. Piskunov ${ }^{1}$, and E. Schilbach ${ }^{3}$ \\ 1 Institute of Astronomy of the Russian Acad. Sci. 48 Pyatnitskaya St., Moscow 109017, Russia \\ e-mail: abelikov@inasan.rssi.ru \\ 2 Main Astronomical Observatory, Golosiiv, 252650 Kiev, Ukraine \\ e-mail: nkhar@mao.kiev.ua \\ 3 Astrophysikalisches Institut Potsdam, An der Sternwarte 16, D-14482 Potsdam, Germany \\ e-mail: eschilbach@aip.de
}

Received May 29; accepted September 18, 1998

\begin{abstract}
A compiled catalogue of astrometric and photometric data for about 2200 stars down to $V=16.8^{\mathrm{m}}$ is presented. The stars are located in an area with a radius of 22.8 arcmin around the extremely young open cluster NGC 6611 with the cluster center at $(\alpha, \delta)_{2000.0}=$ $18^{\mathrm{h}} 18^{\mathrm{m}} 40^{\mathrm{s}},-13^{\circ} 47.1^{\prime}$. The catalogue is based on new reductions of positions, proper motions, and of photometric data that have been published in this region and includes, among others, Hipparcos, AC/GSC and Tautenburg Schmidt plate observations. For the membership determination, spatial and proper motion distributions of stars in the region were analysed. An extinction map of the area and the cluster color-magnitude diagram down to $M_{V}=+1^{\mathrm{m}}$ were constructed. The distance of NGC 6611 was determined as $2.14 \pm 0.10 \mathrm{kpc}$.
\end{abstract}

Key words: open clusters - HR diagram

\section{Introduction}

The open cluster NGC 6611 located near the external border of the Sagittarius-Carina spiral arm $(l, b)=$ $17.0^{\circ},+0.8^{\circ}$ has been a subject of many studies based on astrometric and astrophysical methods. This interest has been caused by the extremely young age of NGC 6611 and by the importance of such investigations for the understanding of star formation and evolution. The cluster is probably associated with the extensive bright emission nebula M 16 (The Eagle Nebula). Morgan et al. (1953)

Send offprint requests to: E. Schilbach

* The complete catalogue is available at the CDS via anonymous ftp to cdsarc.u-strasbg.fr (130.79.128.5) or via http://cdsweb.u-strasbg.fr/Abstract.html assumed NGC 6611 to be physically connected with the association Ser I.

The first extensive photometric study of NGC 6611 was carried out by Walker (1961) from photoelectric and photographic $U B V$ observations of 532 stars down to $V$ $=16.7^{\mathrm{m}}$. He used color excesses of stars as a criterion to pick out cluster members. Hoag et al. (1961) obtained photoelectric $U B V$ photometry of 24 stars down to $V=$ $14.7^{\mathrm{m}}$ in this region. The spectral classification and photoelectric $U B V$ photometry for the 15 brightest stars was carried out by Hiltner \& Morgan (1969). Sagar \& Joshi (1979) determined photoelectric $U B V$ magnitudes for 50 cluster members. The measurements of 89 stars in $U B V R I$ photometric bands were carried out by Thé et al. (1990). Hillenbrand et al. (1993) studied this cluster by combining CCD observations in the $U B V$ system of 1022 stars down to $V=18.7^{\mathrm{m}}$ with spectroscopic and infrared ( $\mathrm{JHK}$ ) photometric data. The CCD observations were also used to derive equatorial coordinates of these stars.

Using plates taken with the $30-\mathrm{cm}$ double refractor of the Observatorium Hoher List, Van Schewick (1962) and Tucholke et al. (1986) determined proper motions of 231 stars down to $B=13^{\mathrm{m}}$ with a baseline of 40 years and of 253 stars down to $B=15.1^{\mathrm{m}}$ with a baseline of 64 years, respectively. Kamp (1974) obtained proper motions for 142 stars down to $V=13.2^{\mathrm{m}}$ with plates taken over 47 years with the Yerkes 40 -inch refractor. In all these studies, proper motion membership probabilities were determined by assuming two different distribution functions for field and cluster stars.

The brightest stars in this region are included in the PPM-South catalogue (Bastian \& Röser 1993). For about 200 stars, proper motions were derived by Röser (1996) from the comparison of positions from the Astrographic Catalogue and the Guide Star Catalog. 
A study of the cluster NGC 6611 based on Schmidt plate observations is given by Kharchenko and Schilbach (1995), hereafter referred to as KS95. Equatorial coordinates, proper motions and photographic $B, V$ magnitudes for about 36000 stars were derived in a field of 8.95 sq. degrees from three plates of the Tautenburg Schmidt telescope $(134 / 203 / 401)$ with a scale of $51.4 \mathrm{arcsec} / \mathrm{mm}$ and a time-span of 24.2 years. The cluster membership probabilities were obtained in the fourdimensional phase space with two components for proper motions and two components for plate coordinates.

On the basis of all these data we compiled an astrometric and photometric survey down to $V=16.8^{\mathrm{m}}$ within a circular area of 0.45 sq. degrees centered at the cluster NGC 6611 . With 2185 entries, the catalogue gives the most representative list of stars in this region and contains equatorial coordinates for the equinox 2000.0 and epoch 1990.77, proper motions in the Hipparcos system as well as optical and infrared photometric data. The catalogue is described in Sect. 2.

Using color excess data gathered in the catalogue, we analyse in Sect. 3 the spatial distribution of the absorption within the cluster, derive the reddening map of this region, construct the reddening-free color-magnitude diagrams, and determine the cluster distance.

\section{Compiled catalogue}

\subsection{Stellar content}

The basic set of 2033 stars down to $V=16.8^{\mathrm{m}}$ was extracted from the Tautenburg survey which provides the most complete list of stars in the NGC 6611 region. The observations, measurements, procedures for proper motion and $B, V$ determination as well as the accuracy of the Tautenburg data are described in KS95. The list was completed by adding 152 bright stars and close optical companions found in the literature. On Schmidt plates, these stars could not be measured properly due to a crowding effect.

Figure 1 shows a map of all 2185 stars included in the compiled catalogue in the NGC 6611 region. The $V$ magnitudes of the stars range from $8.01^{\mathrm{m}}$ to $16.78^{\mathrm{m}}$. The coordinates of the cluster center as determined in this study are $(\alpha, \delta)_{2000.0}=18^{\mathrm{h}} 18^{\mathrm{m}} 40^{\mathrm{s}},-13^{\circ} 47.1^{\prime}$ (see Table 3 ).

\subsection{Coordinates}

Right ascensions and declination $(\alpha, \delta)$ were computed from the plate coordinates measured on the Tautenburg first epoch (1963.45) plate with respect to 51 PPM stars ( $V$ from $6.52^{\mathrm{m}}$ to $10.13^{\mathrm{m}}$ ) uniformly distributed over the field. The solutions were carried out with the least squares
Table 1. Proper motion catalogues used for the construction of the compiled catalogue

\begin{tabular}{lcccc}
\hline Reference & $\begin{array}{c}\text { No. of } \\
\text { stars }\end{array}$ & Band & $\begin{array}{c}\text { Range of } \\
\text { magnitudes }\end{array}$ & $\begin{array}{c}\text { rms error } \\
{[\mathrm{mas} / \mathrm{y}]}\end{array}$ \\
\hline Van Schewick (1962) & 231 & $B$ & $8.4-13.3$ & \pm 3.08 \\
Kamp (1974) & 142 & $V$ & $9.4-13.7$ & \pm 1.07 \\
Tucholke et al. (1986) & 233 & $B$ & $8.6-15.5$ & \pm 1.55 \\
PPM-South & 10 & $V$ & $8.1-9.7$ & \pm 2.6 \\
KS95 & 2564 & $B, V$ & $8.0-16.8$ & see text \\
Röser (1996) & 210 & $B$ & $7.85-13.4$ & \pm 4.1 \\
H/KS & 1022 & $B, V$ & $7.95-18.7$ & \pm 4.6 \\
\hline
\end{tabular}

technique applied to polynomials

$$
\begin{aligned}
& \xi-X=Q_{a}(X, Y)+a_{1} X\left(X^{2}+Y^{2}\right)+a_{2} B, \\
& \eta-Y=Q_{b}(X, Y)+b_{1} Y\left(X^{2}+Y^{2}\right)+b_{2} B .
\end{aligned}
$$

Here $B$ stands for a stellar magnitude, $\xi, \eta$ are tangential coordinates with respect to the plate center and $Q(X, Y)$ is a complete 2-nd order polynomial. We determined a magnitude equation in the $Y$ coordinate as $b_{2}=(22 \pm 5) 10^{-4} \mathrm{~mm} / \mathrm{mag}$ and the distortion terms due to the curvature of the Schmidt plate as $a_{1}=(2.05 \pm 0.20) 10^{-8} \mathrm{~mm}^{-2}$ and $b_{1}=$ (1.41 \pm 0.18$) 10^{-8} \mathrm{~mm}^{-2}$. Coefficient $a_{2}$ is found to be insignificant. The accuracy of the right ascension and declination at the epoch 1963.45 is $0.008 \mathrm{~s}$ and 0.14 arcsec, respectively. For the final version of the catalogue, the coordinates were recomputed for the equinox 2000.0 and epoch 1990.77.

For 99 stars not measured on the Tautenburg plates, the positions were taken from Hillenbrand et al. (1993) where the equatorial coordinates were derived for the equinox 2000.0 with the Guide Star Catalog (GSC) as a reference. 53 stars of Walker (1961) could not be found either in the Tautenburg survey (KS95) or in the list of Hillenbrand et al. (1993). For these stars, only their approximate rectangular coordinates taken from Walker's (1961) maps are given in the catalogue.

\subsection{Absolute proper motions}

\subsubsection{Tautenburg proper motion survey (KS95)}

Relative proper motions were derived from the measurements of the Tautenburg plates with respect to a large sample of anonymous stars (some 13000 stars) selected among field stars with small proper motions. The proper motions of stars with the highest probability to belong to one of the four open clusters (Trümpler 32, NGC 6611, C 1819-146, C 1820-146) identified on the plates were used to correct the proper motions of all other stars for a magnitude-dependent error (see KS95).

The accuracy of the Tautenburg proper motions was estimated as 2.7 mas/year from a comparison of our data 


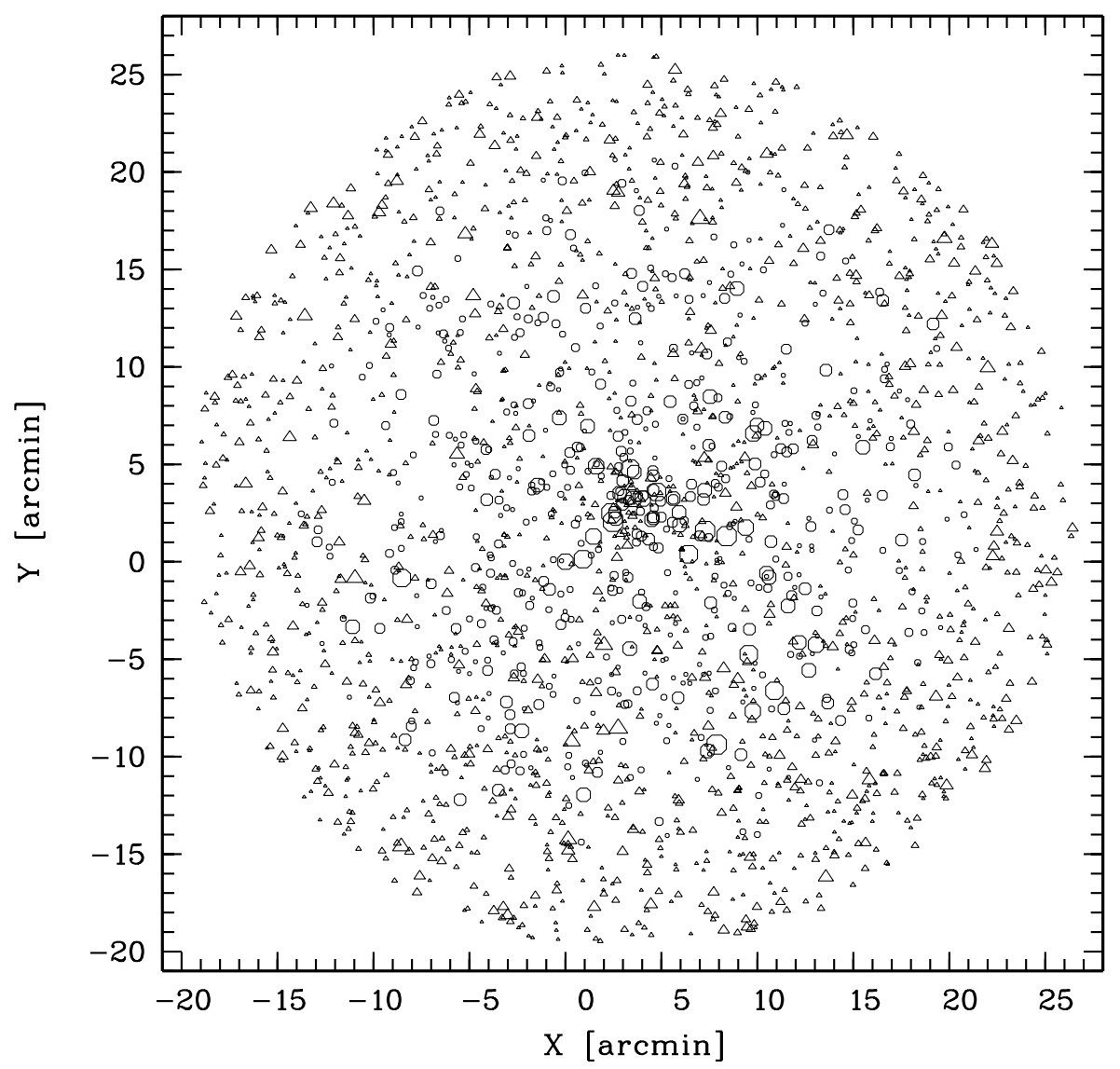

Fig. 1. Distribution of field stars (triangles) and the cluster members (open circles) of the survey within the NGC 6611 region. The size of symbols corresponds to the brightness of stars. The magnitude range is $\Delta V=8.01-16.78^{\mathrm{m}}$. The coordinates $X, Y$ are given in arcmin with respect to the Walker's star $125(\alpha, \delta)_{2000.0}=18^{\mathrm{h}} 18^{\mathrm{m}} 26.21^{\mathrm{s}},-13^{\circ} 50^{\prime} 05.3^{\prime \prime}$ and increase with right ascension $(X)$ and declination $(Y)$

with the results published by Van Schewick (1962), Kamp (1974) and Tucholke et al. (1986) for the stars in common $\left(B=9.8^{\mathrm{m}}-14.6^{\mathrm{m}}\right)$. Outside of this magnitude range the proper motion accuracy is lower and decreases rapidly for stars fainter than $B=16^{\mathrm{m}}$. A dependence of the proper motion rms error $\varepsilon_{\mu}$ on magnitudes within $B=7^{\mathrm{m}}-17^{\mathrm{m}}$ was found to be best fitted by a polynomial

$$
\begin{aligned}
\varepsilon_{\mu}[\mathrm{mas} / \mathrm{y}]= & 0.0101(B-13)^{4}+0.051(B-13)^{3} \\
& -0.4478(B-13)+2.6832 .
\end{aligned}
$$

Since no galaxies could be found in this direction of the sky, we originally used PPM-South stars (Bastian \& Röser 1993) in KS95 to convert the relative proper motions from the Tautenburg survey to absolute ones. Meanwhile, the Hipparcos catalogue (ESA 1997) is available, so we recomputed the zero point corrections for the Tautenburg proper motions with respect to the Hipparcos reference system. Although 22 Hipparcos stars were identified in the Tautenburg survey covering a field of 8.95 sq. degrees, one half of them was too bright to be properly measured on Schmidt plates. The zero point corrections for the Tautenburg proper motions with respect to the Hipparcos reference system were computed to be

$$
\begin{aligned}
& \overline{\mu_{X}-\mu_{X}^{\mathrm{HIPP}}}=-0.4 \pm 1.5 \mathrm{mas} / \mathrm{yr}, \\
& \overline{\mu_{Y}-\mu_{Y}^{\mathrm{HIPP}}}=+1.1 \pm 0.95 \mathrm{mas} / \mathrm{yr} .
\end{aligned}
$$

After the Tautenburg data were corrected for the zero point and magnitude-dependent errors, we assume KS95 to represent the proper motion system on which the compiled catalogue is now based.

2.3.2. Proper motions $(\mathrm{H} / \mathrm{KS})$ for Hillenbrand et al. (1993) stars

Whereas proper motions for the bright stars in the NGC 6611 region were determined by several authors, there are only two studies, KS95 and Hillenbrand et al. (1993), where astrometric information for stars fainter than $V=15^{\mathrm{m}}$ is presented. That was the main reason to include CCD-positions obtained by Hillenbrand et al. (1993) in our catalogue. In their paper the authors listed 
the equatorial coordinates of about 1000 stars for the equinox 2000.0 and epoch 1990.77. The coordinates were derived in the system of the GSC. The accuracy of positions was estimated by the authors to be 0.2 arcsec. Proper motions were derived by combining these positions with the coordinates at the epoch 1963.45 computed according to Eq. (1) from measurements of the first epoch plate of the Tautenburg survey. The sample is marked by $\mathrm{H} / \mathrm{KS}$ in Table 1. Taking into account the accuracy of the Tautenburg and CCD-positions and a time-span of 27.32 years, a formal error of 9 mas/yr may be expected for these proper motions. Nevertheless, from a comparison of the proper motions from $\mathrm{H} / \mathrm{KS}$ with the data of Van Schewick (1962), Kamp (1974) and Tucholke et al. (1986) an accuracy of $4.6 \mathrm{mas} / \mathrm{yr}$ was estimated for the stars in common $\left(B=8.0^{\mathrm{m}}-14.6^{\mathrm{m}}\right)$. This result indicates that at least for these stars the errors seem to be overestimated both for the Tautenburg and CCD-positions from Hillenbrand et al. (1993). For the Tautenburg survey this conclusion seems to be justified because the error of $0.12-0.14$ arcsec given in Chapter 2.2 for the coordinates refers to stars distributed over the whole field covered by a Tautenburg plate (c.a. 9 sq.degrees) whereas the cluster NGC 6611 is located close to the plate center. As in Hillenbrand et al. (1993) the coordinates were used exclusively for the identification reasons, the authors gave only a brief description of the atrometric reduction applied. In any case, we may conclude that the accuracy of the Hillenbrand et al. (1993) coordinates should be better than the authors assumed.

Using the data from KS95 and $\mathrm{H} / \mathrm{KS}$, one has to take into account that the proper motions are highly correlated since they are based on the same first epoch observations. They differ due to the second epoch observations and the reduction method applied. The Tautenburg proper motions were derived by a plate-to-plate reduction with a time baseline of 24.2 years. The $\mathrm{H} / \mathrm{KS}$ proper motions were computed from differences of equatorial coordinates for the equinox 2000.0 over a time-span of 27.32 years.

\subsubsection{Compilation of the proper motions}

In order to derive proper motions in a common system, we applied a method which is generally used for the construction of compilation catalogues (e.g., Eichhorn 1974). Each proper motion catalogue from Table 1 was reduced to the system of the Tautenburg survey (KS95) by correction for different zero points and magnitude-dependent errors.

In order to determine weights for each catalogue, we used about 250 stars with at least three independent proper motion determinations (note: the catalogue pairs KS95 and H/KS as well as Van Schewick (1962) and Tucholke et al. (1986) were considered as correlated catalogues). From proper motion residuals of the same stars included in three catalogues, external rms errors of each
Table 2. Photometric catalogues used for the construction of the compiled catalogue

\begin{tabular}{lcccc}
\hline Reference & \multicolumn{3}{c}{$\begin{array}{c}\text { Method No. of } \\
\text { stars }\end{array}$} & $\begin{array}{c}\text { Range of } V \\
\text { magnitude }\end{array}$ \\
\hline Walker (1961) & photoel. & 55 & 75 & $8.24-16.75$ \\
Walker (1961) & photogr. & 536 & & $9.78-16.84$ \\
Hoag et al. (1961) & photoel. & 24 & - & $8.19-14.69$ \\
Hiltner \& Morgan (1969) & photoel. & 15 & 15 & $8.24-11.18$ \\
Sagar \& Joshi (1979) & photoel. & 50 & 50 & $8.21-13.29$ \\
Thé et al. (1990) & photoel. & 89 & 52 & $8.20-15.07$ \\
Hillenbrand et al. (1993) & CCD & 1022 & - & $7.95-18.7$ \\
KS95 & photogr. & 2564 & - & $8.0-16.8$ \\
\hline
\end{tabular}

catalogue could be estimated (see KS95). The corresponding rms errors which were used to define weights of the catalogues are given in the last column of Table 1 . These weights were used in computing the mean proper motions and the corresponding rms errors for stars of the compiled catalogue. For the stars whose proper motions were obtained with the Tautenburg plates only, the rms errors were computed according to Eq. (2). Figure 2 shows the differences of proper motions from the compiled catalogue and the source catalogues. Only those stars are plotted which appear in at least two catalogues listed in Table 1.

In total, our catalogue contains proper motions for 2074 stars. To check these proper motions for possible magnitude-dependent errors, we used data on cluster members with the assumption that the proper motions of cluster stars are independent of the apparent magnitude. Figure 3 shows absolute proper motions versus stellar magnitude $V$ for all 2074 stars and for probable cluster members. According to Fig. 3, we can conclude that the proper motions are free from magnitude-dependent errors both in $X$ and $Y$ directions.

\subsection{Photometric data}

The calibration of $B$ and $V$ Tautenburg plates in KS95 was based on photoelectric sequences taken from Walker (1961), Sagar \& Joshi (1979), and Nicolet (1978). The background effect due to M 16 was taken into account in photographic magnitudes by a term

$k \cdot \exp \left[-0.5 \cdot\left[(X-\bar{X})^{2} / \sigma_{X}^{2}+(Y-\bar{Y})^{2} / \sigma_{Y}^{2}\right]\right]$

where $\bar{X}, \bar{Y}$ are the coordinates of the centre of M 16 . For the factor $k$ values of $0.25^{\mathrm{m}} \pm 0.06,0.32^{\mathrm{m}} \pm 0.04$ and $0.15^{\mathrm{m}} \pm 0.04$ were obtained for two $B$ plates and one $V$ plate, respectively. The accuracy of the photometric data was estimated as $\sigma_{B}= \pm 0.12^{\mathrm{m}}$ and $\sigma_{V}= \pm 0.10^{\mathrm{m}}$ (see KS95).

In addition, we included the relevant data from the photometric catalogues obtained in the NGC 6611 region (see Table 2). 

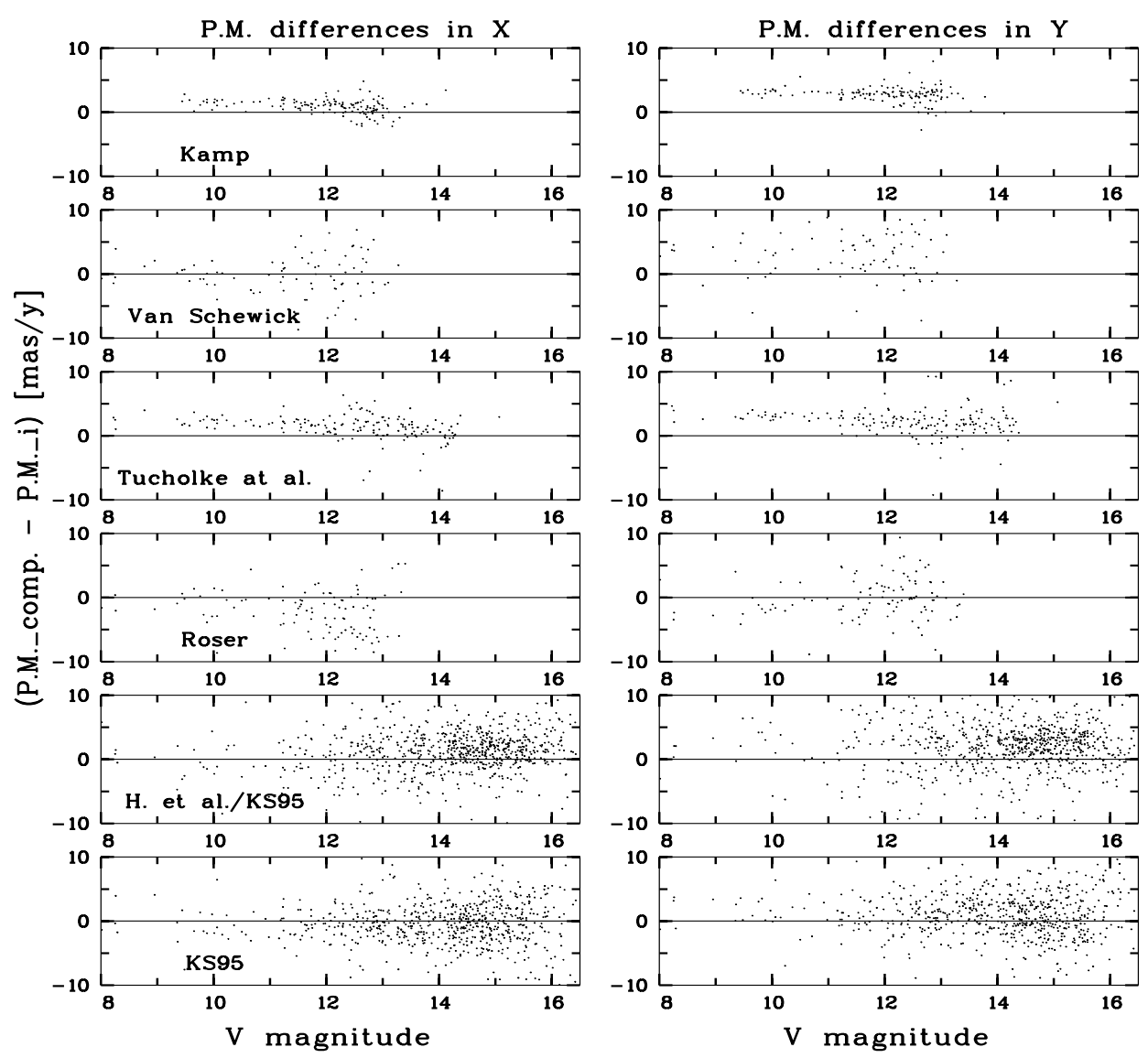

Fig. 2. Differences in proper motions between the compiled and the original catalogues (see Table 1 ) as a function of $V$
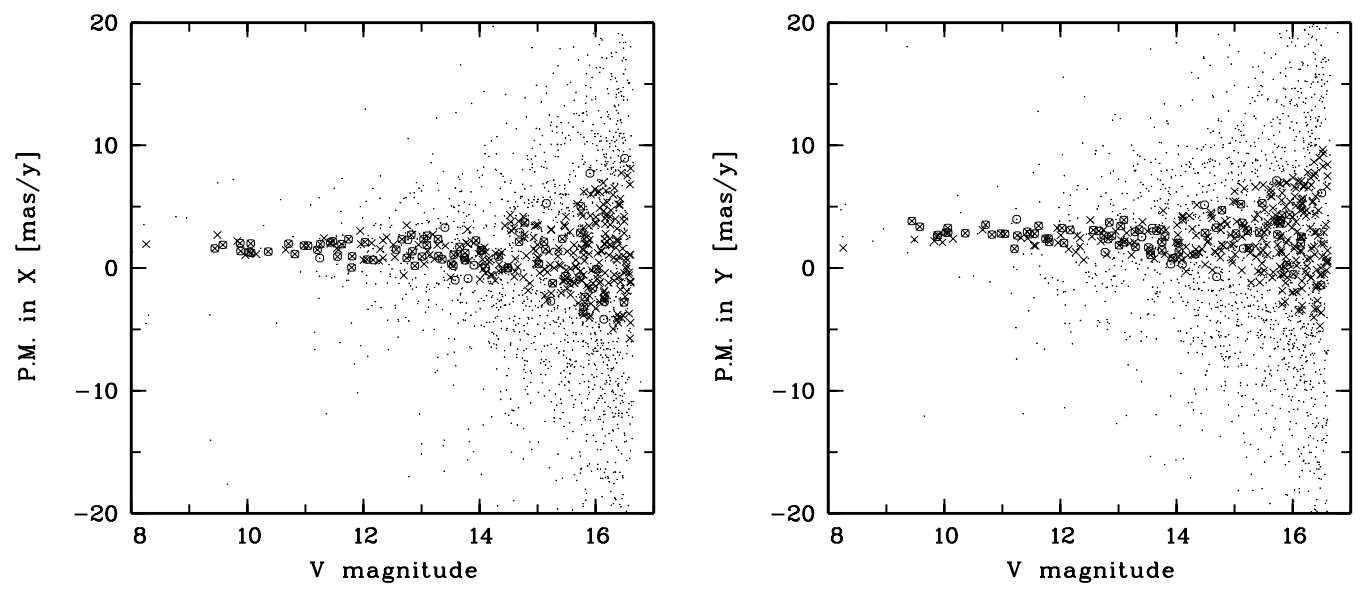

Fig. 3. Absolute proper motion components in $X$ and $Y$ directions versus $V$ magnitude. Dots: all stars included in the compiled catalogue; open circles: stars with the highest membership probability based both on proper motions and coordinates; crosses: stars with the highest membership probability based on proper motions only 

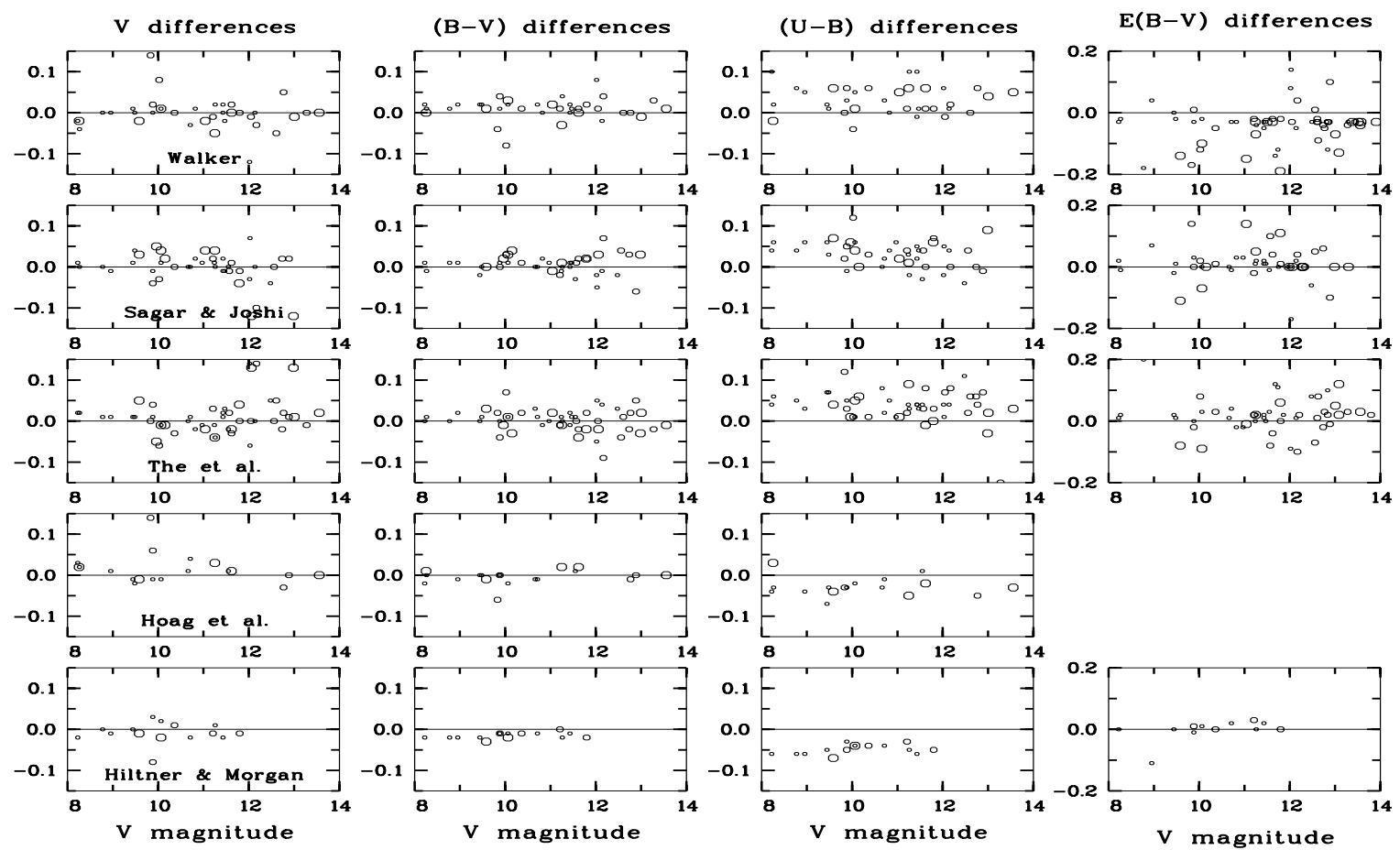

Fig. 4. Differences in magnitudes, colors and color excesses between the compiled catalogue and the original photoelectric data (see Table 2) versus $V$ magnitudes. Small, medium and large circles indicate stars with $(B-V) \leq 0.5^{\mathrm{m}}, 0.5<(B-V) \leq 0.8^{\mathrm{m}}$ and $(B-V)>0.8^{\mathrm{m}}$, respectively
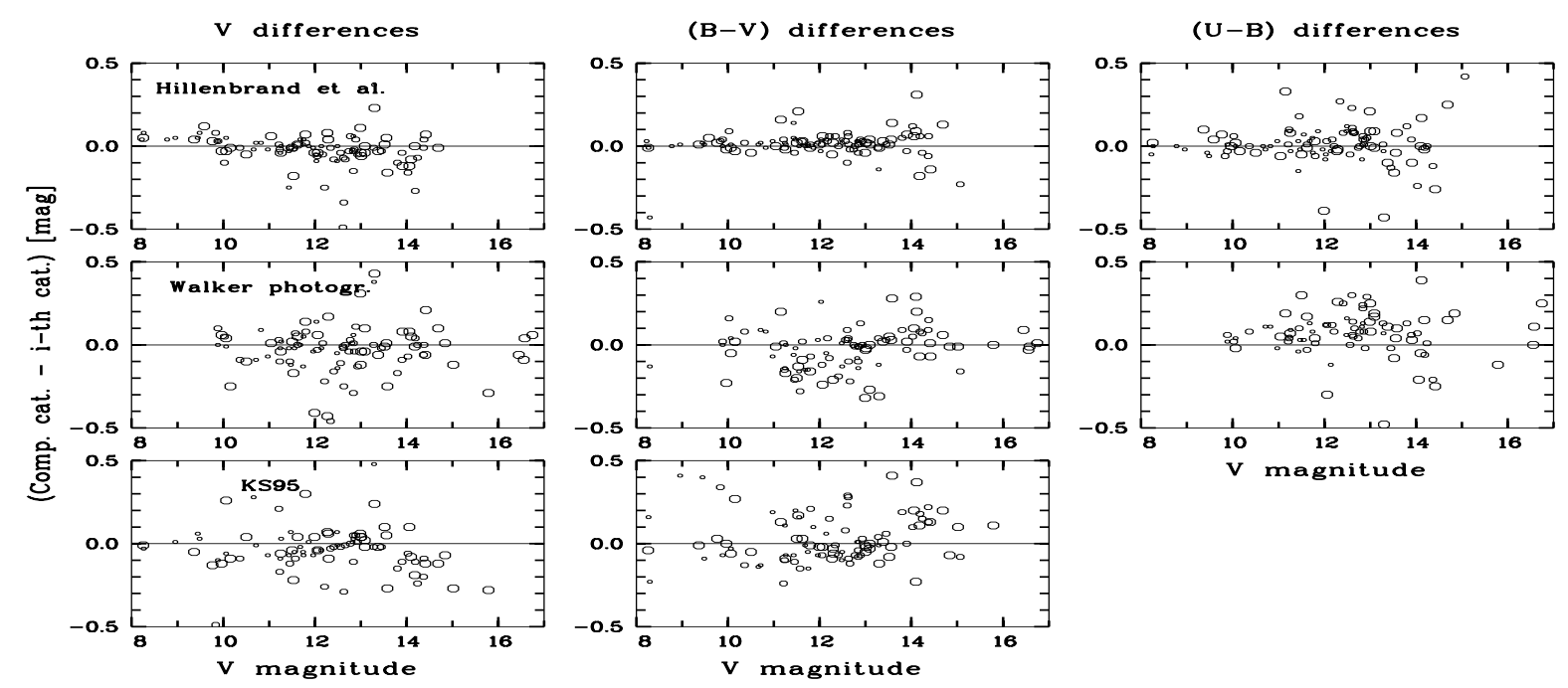

Fig. 5. Differences in magnitudes and colors between the compiled catalogue and original CCD and photographic data (see Table 2). Small, medium and large open circles indicate the stars with $(B-V) \leq 0.5^{\mathrm{m}}, 0.5<(B-V) \leq 0.8^{\mathrm{m}}$ and $(B-V)>0.8^{\mathrm{m}}$, respectively. Only stars with photoelectrically determined magnitudes and colors are plotted 
The photometric $U B V$ system of the compiled catalogue is defined by the common photoelectric sequences described below. Since the accuracy of the photoelectric data achieved by the authors from Table 2 is comparable, we assumed equal weights for all photoelectric catalogues. However, from a comparison of magnitudes for stars in common, we found systematic differences between the data of Walker (1961), Sagar \& Joshi (1979), Thé et al. (1990) on one side and Hoag et al. (1961), Hiltner \& Morgan (1969) on the other side. Especially, for $U$ magnitudes this effect was highly significant (see Fig. 4). Therefore, the photometric reduction was performed in three steps: for each of the two groups, we derived its own photoelectric system; the common photoelectric system was defined as the mean of the intermediate systems; finally, the corresponding zero point correction was introduced in the original magnitude for each star. A similar procedure was applied to the original color excesses $E_{(B-V)}$ to combine them into a common system. Our catalogue includes $121,118,115$ and 97 stars with photoelectric $V$ magnitudes, $(B-V),(U-B)$ and color excesses $E_{(B-V)}$, respectively.

For stars without photoelectric data, magnitudes and colors in our catalogue are based on CCD and photographic measurements. A deep CCD photometry was obtained in the NGC 6611 region by Hillenbrand et al. (1993). An analysis of these data (Fig. 5) showed that they differed systematically by a few $0.01^{\mathrm{m}}$ from the compiled photoelectric system which we assumed to be the most reliable.

From a comparison of the photoelectric and CCD sequences, we computed the corrections to $U B V_{\mathrm{CCD}}$ magnitudes of Hillenbrand et al. (1993) as

$U_{\text {photoel. }}-U_{\mathrm{CCD}}=0.201^{\mathrm{m}}-0.0157 U_{\mathrm{CCD}}$,

$B_{\text {photoel. }}-B_{\mathrm{CCD}}=0.185^{\mathrm{m}}-0.0146 B_{\mathrm{CCD}}$,

$V_{\text {photoel. }}-V_{\mathrm{CCD}}=0.184^{\mathrm{m}}-0.0165 V_{\mathrm{CCD}}$.

The photographic data from KS95 and Walker (1961) were also reduced to the compiled photoelectric system. The $U, B, V$ magnitudes of stars without photoelectric photometry were computed as weighted mean values of the reduced CCD and photographic data. Weights for the CCD data were determined from rms errors given in Hillenbrand et al. (1993) as a function of the $V$ magnitudes (their Fig. 3). For photographic data, much higher rms errors, $\sigma_{V}= \pm 0.10^{\mathrm{m}}, \sigma_{B}= \pm 0.12^{\mathrm{m}}$, and $\sigma_{U}= \pm 0.15^{\mathrm{m}}$, were assumed.

Thus all $U B V$ photometric data were derived into a common system based on the photoelectric sequence described above. Totally, our catalogue includes 2185 stars with $V$ magnitudes and $(B-V)$ color indexes, 917 stars with $(U-B)$ color indexes and 97 stars with $E_{(B-V)}$ color excesses. For completeness, the original infrared $J H K$ photometry obtained by Hillenbrand et al. (1993) from
Table 3. Parameters of the distribution functions in the NGC 6611 region

\begin{tabular}{ccc}
\hline Parameter & Units & \\
\hline $\bar{X}_{\mathrm{cl}}$ & {$[$ arcmin $]$} & +3.5 \\
$\bar{Y}_{\mathrm{cl}}$ & {$[$ arcmin $]$} & +3.0 \\
$\sigma_{X Y}^{\text {core }}$ & {$[$ arcmin $]$} & 1.20 \\
$\sigma_{X Y}^{\text {corona }}$ & {$[$ arcmin $]$} & 4.77 \\
$\bar{\mu}_{X \mathrm{cl}}$ & {$[$ mas/year $]$} & +1.42 \\
$\bar{\mu}_{Y \mathrm{cl}}$ & {$[$ mas/year $]$} & +2.02 \\
$\bar{\mu}_{X \mathrm{df}}$ & {$[$ mas/year $]$} & -0.07 \\
$\bar{\mu}_{Y \mathrm{df}}$ & {$[$ mas/year $]$} & +1.68 \\
$\bar{\mu}_{X \mathrm{nf}}$ & {$[$ mas/year $]$} & -4.75 \\
$\bar{\mu}_{Y \mathrm{nf}}$ & {$[$ mas/year $]$} & +1.92 \\
$\sigma_{\mu}^{X \mathrm{cl} Y \mathrm{cl}}$ & {$[$ mas/year $]$} & 1.52 \\
$\sigma_{\mu}^{X \mathrm{df} Y \mathrm{df}}$ & {$[$ mas/year $]$} & 6.13 \\
$\sigma_{\mu}^{X \mathrm{nf} Y \mathrm{nf}}$ & {$[$ mas/year $]$} & 14.04 \\
$n_{\mathrm{cl}}$ & {$[$ per cent $]$} & 17 \\
$n_{\mathrm{df}}$ & {$[$ per cent $]$} & 68 \\
$n_{\mathrm{nf}}$ & {$[$ per cent $]$} & 14 \\
\hline & &
\end{tabular}

CCD observations are also given in the compiled catalogue (384 stars).

\subsection{Cluster membership probabilities}

For such distant clusters as NGC 6611, the extraction of members from proper motions only is rather uncertain due the low significance of the differences between individual proper motions of cluster members and distant field stars. On the other hand, distant clusters show a local concentration of stars in the projection on the sky which provides an additional criterion for the selection of members.

In order to improve the reliability of membership determination, we applied a statistical method described in KS95 and Kharchenko et al. (1997) which used the information both position and proper motion distributions of stars in the cluster neighbourhood.

The distribution function $F\left(\mu_{X}, \mu_{Y}, X, Y\right)$ of stars in the vicinity of a cluster is assumed to be the sum of two distribution functions of field and cluster stars, respectively:

$F\left(\mu_{X}, \mu_{Y}, X, Y\right)=F_{\mathrm{f}}\left(\mu_{X}, \mu_{Y}, X, Y\right)+F_{\mathrm{cl}}\left(\mu_{X}, \mu_{Y}, X, Y\right)$.

The cluster distribution itself can be divided in two components, the core and corona distributions which have different spatial dispersions. The field stellar population is 
Table 4. Distribution of the color excesses $E_{(B-V)}$ (top) and $R_{V}$ values (bottom) over the NGC 6611 region. The coordinates of the cluster centre are $(X, Y)=+3.5,+3.0$ which correspond to $(\alpha, \delta)_{2000.0}=18^{\mathrm{h}} 18^{\mathrm{m}} 40^{\mathrm{s}},-13^{\circ} 47.1^{\prime}$

\begin{tabular}{|c|c|c|c|c|c|c|c|c|c|c|c|c|c|c|c|c|c|c|c|c|c|}
\hline \multirow{2}{*}{$\begin{array}{c}Y, \\
\operatorname{arcmin}\end{array}$} & \multicolumn{21}{|c|}{$X$, arcmin } \\
\hline & -17 & -15 & -13 & -11 & -9 & -7 & -5 & -3 & -1 & 1 & 3 & 5 & 7 & 9 & 11 & 13 & 15 & 17 & 19 & 21 & 23 \\
\hline-15 & $=$ & - & - & - & $=$ & - & - & - & - & - & - & 二 & - & - & $\underline{0.77}$ & - & $=$ & - & - & - & - \\
\hline \multirow{2}{*}{-13} & & - & - & - & - & - & 0.58 & - & - & 1.13 & - & - & - & 0.75 & - & - & 0.76 & - & - & - & - \\
\hline & $\overline{-}$ & $\overline{-}$ & $\overline{-}$ & $\overline{-}$ & $\overline{-}$ & $\overline{-}$ & 3.97 & $\overline{-}$ & $\overline{-}$ & $\overline{-}$ & $\overline{-}$ & $\overline{-}$ & $\overline{-}$ & - & $\overline{-}$ & $\overline{-}$ & $\overline{-}$ & $\overline{-}$ & $\overline{-}$ & $=$ & $\overline{-}$ \\
\hline \multirow{2}{*}{-11} & 二 & - & 二 & $\bar{z}$ & - & - & - & 0.81 & $\overline{-}$ & 0.62 & 0.98 & - & - & \begin{tabular}{|l|l|}
0.84 \\
\end{tabular} & 0.85 & - & 0.62 & - & - & - & $\overline{-}$ \\
\hline & $=$ & $\overline{-}$ & $=$ & $\overline{-}$ & $=$ & $\overline{-}$ & $\overline{-}$ & $\overline{-}$ & $\overline{-}$ & - & $\overline{-}$ & $=$ & $\overline{-}$ & - & - & $\overline{-}$ & 3.10 & $\overline{-}$ & $\overline{-}$ & $=$ & $=$ \\
\hline \multirow{2}{*}{-9} & - & - & - & - & - & - & - & 0.69 & - & - & 0.81 & 0.74 & 0.63 & - & - & - & 0.81 & - & 0.68 & - & - \\
\hline & $\overline{-}$ & $\overline{-}$ & $\overline{-}$ & $\overline{-}$ & $\overline{-}$ & $\overline{-}$ & $=$ & - & $\overline{-}$ & $\overline{-}$ & - & - & $\overline{3.19}$ & $\overline{-}$ & $\overline{-}$ & $\overline{-}$ & $\overline{-}$ & $=$ & - & $=$ & $\overline{-}$ \\
\hline \multirow{2}{*}{-7} & 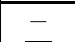 & - & - & - & - & & - & 0.54 & - & - & 0.80 & 0.53 & \begin{tabular}{|l|}
0.97 \\
\end{tabular} & 0.63 & 0.67 & 0.58 & - & - & - & - & 二 \\
\hline & $=$ & $\overline{-}$ & $\overline{-}$ & $\overline{-}$ & $\overline{-}$ & $\overline{-}$ & $\overline{-}$ & $\overline{-}$ & $\overline{-}$ & $\overline{-}$ & $\overline{-}$ & $\overline{3.80}$ & - & $\overline{3.16}$ & $\overline{4.42}$ & - & $\overline{-}$ & $\overline{-}$ & $\overline{-}$ & $=$ & $\overline{-}$ \\
\hline \multirow{2}{*}{-5} & 二 & 二 & $\bar{z}$ & - & - & - & 0.68 & - & - & 1.04 & 0.78 & 1.08 & \begin{tabular}{|l|}
1.07 \\
\end{tabular} & 0.57 & 1.34 & 0.61 & 1.04 & 0.93 & 0.84 & - & - \\
\hline & $=$ & $\overline{-}$ & $=$ & $=$ & $=$ & $=$ & - & $=$ & $\overline{-}$ & $\overline{3.86}$ & $\overline{3.19}$ & $\overline{3.29}$ & - & $\overline{3.38}$ & - & $\overline{3.50}$ & - & - & - & $=$ & $=$ \\
\hline \multirow{2}{*}{-3} & 1.28 & - & 0.63 & 0.72 & 0.56 & - & \begin{tabular}{|l|}
0.98 \\
\end{tabular} & 1.05 & \begin{tabular}{|l|}
0.59 \\
\end{tabular} & - & 1.27 & \begin{tabular}{|l}
1.18 \\
\end{tabular} & \begin{tabular}{|l|}
0.77 \\
\end{tabular} & \begin{tabular}{|l|}
0.53 \\
\end{tabular} & \begin{tabular}{|l|}
0.70 \\
\end{tabular} & \begin{tabular}{|l|l}
0.60 \\
\end{tabular} & \begin{tabular}{|l|l}
1.18 \\
\end{tabular} & - & - & - & 0.68 \\
\hline & $7-$ & $\overline{-}$ & $\overline{-}$ & $\overline{3.40}$ & $\overline{4.35}$ & $\overline{-}$ & $\overline{2.80}$ & $\overline{3.74}$ & $\overline{3.90}$ & $=$ & $\overline{2.90}$ & $\overline{3.14}$ & $\overline{2.49}$ & - & $\overline{3.10}$ & - & - & $=$ & $\overline{-}$ & $=$ & - \\
\hline \multirow[b]{2}{*}{-1} & 1.29 & 1.04 & - & 0.83 & 1.00 & - & 1.51 & - & \begin{tabular}{|l|l|}
1.14 \\
\end{tabular} & 1.35 & 0.81 & - & \begin{tabular}{|l|}
- \\
\end{tabular} & \begin{tabular}{|l|}
0.95 \\
\end{tabular} & 1.00 & \begin{tabular}{|l|l|} 
\\
\end{tabular} & 0.85 & 1.12 & - & - & - \\
\hline & $\overline{-}$ & - & $\overline{-}$ & $\overline{-}$ & $\overline{3.30}$ & $\overline{-}$ & 3.47 & $=$ & $\overline{2.92}$ & $\overline{2.86}$ & $\overline{3.51}$ & $=$ & $=$ & $\overline{3.11}$ & - & $\overline{3.10}$ & - & $\overline{-}$ & $=$ & $=$ & $\overline{-}$ \\
\hline \multirow{2}{*}{1} & $\overline{-}$ & 二 & $\overline{-}$ & $\bar{z}$ & 1.71 & $\overline{-}$ & $\bar{z}$ & $\bar{z}$ & 二- & 0.86 & 0.71 & 0.88 & \begin{tabular}{|l|}
0.69 \\
\end{tabular} & 0.86 & - & 0.65 & 0.76 & 二 & 二 & 二 & 二 \\
\hline & $\overline{-}$ & $=$ & $=$ & $=$ & $\overline{-}$ & $\overline{-}$ & $=$ & $=$ & $\overline{-}$ & \begin{tabular}{|l|}
3.43 \\
\end{tabular} & 3.51 & 3.47 & \begin{tabular}{|l|}
3.45 \\
\end{tabular} & $\overline{3.40}$ & $\overline{-}$ & $\overline{-}$ & - & $\overline{-}$ & $\overline{-}$ & $=$ & $\overline{-}$ \\
\hline \multirow[b]{2}{*}{3} & - & 二 & - & 二 & 二 & - & 1.26 & 二 & 0.86 & - & 0.77 & 0.82 & \begin{tabular}{|l|}
0.75 \\
\end{tabular} & - & 0.87 & - & 0.78 & - & - & - & - \\
\hline & $=$ & $\overline{-}$ & $\overline{-}$ & $\overline{-}$ & $\overline{-}$ & $\overline{-}$ & 3.70 & $=$ & $\overline{3.23}$ & $\overline{-}$ & 3.68 & $\overline{3.61}$ & 3.58 & $\overline{-}$ & $\overline{-}$ & $\overline{-}$ & - & $\overline{-}$ & $\overline{-}$ & $=$ & $\overline{-}$ \\
\hline & $\begin{array}{l}0.60 \\
\end{array}$ & - & - & - & - & - & 0.68 & 0.99 & 1.13 & 0.92 & 1.10 & 1.16 & \begin{tabular}{|l|}
0.62 \\
\end{tabular} & 0.63 & 0.61 & - & \begin{tabular}{|l|}
0.62 \\
\end{tabular} & \begin{tabular}{|l|}
0.72 \\
\end{tabular} & \begin{tabular}{|l|}
0.62 \\
\end{tabular} & - & - \\
\hline & $\overline{-}$ & $=$ & $\overline{-}$ & $\overline{-}$ & $\bar{E}$ & $\overline{-}$ & 3.37 & 4.50 & $\overline{3.59}$ & 3.70 & 3.56 & $\overline{3.62}$ & $\overline{3.10}$ & $\overline{3.10}$ & $\overline{3.10}$ & $\overline{-}$ & $\overline{3.10}$ & - & - & $=$ & $\overline{-}$ \\
\hline \multirow{2}{*}{7} & - & $\overline{-}$ & - & $\overline{-}$ & - & $\overline{-}$ & 1.38 & 1.74 & 0.68 & 1.38 & 1.49 & 0.84 & 0.66 & \begin{tabular}{|l|l|}
0.83 \\
\end{tabular} & 0.92 & \begin{tabular}{|l|l|}
0.83 \\
\end{tabular} & - & - & - & - & - \\
\hline & $=$ & $=$ & $\overline{-}$ & $=$ & $\overline{-}$ & $\overline{-}$ & \begin{tabular}{|l|}
2.83 \\
\end{tabular} & \begin{tabular}{|l|}
2.63 \\
\end{tabular} & $\overline{2.90}$ & $\overline{3.94}$ & 3.51 & $\overline{2.75}$ & $\overline{4.51}$ & 3.45 & $\overline{3.10}$ & - & $\bar{E}$ & $\overline{-}$ & $\overline{-}$ & $\overline{-}$ & $\overline{-}$ \\
\hline & - & 二 & - & - & 0.65 & - & - & 1.18 & - & 1.71 & - & - & 二 & $=$ & 1.56 & 0.70 & - & - & - & - & - \\
\hline & $\overline{-}$ & $\bar{z}$ & $\overline{-}$ & $=$ & 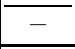 & $\bar{z}$ & $\overline{-}$ & $\overline{-}$ & $\overline{-}$ & $\overline{3.30}$ & $\overline{-}$ & $\overline{-}$ & $\overline{-}$ & $\overline{-}$ & \begin{tabular}{|l|}
3.53 \\
\end{tabular} & $\overline{2.99}$ & $\overline{-}$ & $=$ & $\overline{-}$ & $=$ & $\overline{-}$ \\
\hline \multirow[t]{2}{*}{11} & - & - & $\overline{-}$ & 1.14 & 二 & 0.83 & 二 & 二 & 二 & $=$ & 1.02 & - & $\overline{-}$ & 1.18 & - & - & - & $\overline{-}$ & 0.62 & - & $\bar{z}$ \\
\hline & & & & & & & & - & & & - & - & & - & - & - & - & - & - & - & - \\
\hline \multirow[t]{2}{*}{13} & - & - & - & - & 0.84 & - & - & 0.54 & 1.33 & - & - & 1.31 & - & 0.73 & - & 1.12 & - & 1.09 & \begin{tabular}{|l|} 
\\
\end{tabular} & - & 1.01 \\
\hline & - & - & - & - & - & - & - & - & - & - & - & - & - & - & - & - & - & - & \begin{tabular}{|l|}
3.10 \\
\end{tabular} & - & - \\
\hline \multirow[t]{2}{*}{1} & - & 二 & - & - & - & - & 二 & - & & & 二 & 0.72 & \begin{tabular}{|l|}
0.84 \\
\end{tabular} & \begin{tabular}{|l|l}
1.17 \\
\end{tabular} & - & - & 0.62 & \begin{tabular}{|l|}
1.06 \\
\end{tabular} & - & - & 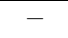 \\
\hline & $\overline{-}$ & $\overline{-}$ & $\overline{-}$ & $\overline{-}$ & $\overline{-}$ & - & $\overline{-}$ & $\overline{-}$ & $\overline{-}$ & $\overline{-}$ & $\overline{-}$ & $\overline{-}$ & $\overline{-}$ & - & $\overline{-}$ & $\overline{-}$ & $\overline{3.10}$ & - & $\overline{-}$ & $\overline{-}$ & $\overline{-}$ \\
\hline \multirow{2}{*}{17} & - & - & 二 & $\overline{-}$ & $\overline{-}$ & - & - & - & - & - & - & - & - & - & 二 & 0.79 & 0.84 & - & - & - & 二 \\
\hline & - & - & - & - & - & - & - & - & - & - & - & - & - & - & - & - & - & - & - & - & - \\
\hline 19 & 二 & $\frac{-}{-}$ & $\frac{-}{二}$ & - & $\overline{-}$ & - & - & $\overline{-}$ & $\underline{0.82}$ & $\underline{-}$ & $\frac{-}{-}$ & $\underline{1.38}$ & $\frac{-}{-}$ & $\underline{-}$ & $\underline{-}$ & $\frac{-}{-}$ & $\frac{-}{-}$ & $\frac{0.89}{-}$ & $=$ & 二 & $=$ \\
\hline 21 & - & - & 二 & - & - & 二 & - & 二 & - & 二 & - & - & 0.85 & $\underline{-}$ & 0.82 & 0.53 & - & - & - & - & - \\
\hline
\end{tabular}

not homogeneous also, since field stars being observed at different distances have different kinematical properties. Therefore, the distribution function of proper motions of field stars can be regarded again as the sum of at least two components (near and distant). So we get $F=F_{\mathrm{f}}+F_{\mathrm{cl}}=F_{\mathrm{f}}^{\text {near }}+F_{\mathrm{f}}^{\text {distant }}+F_{\mathrm{cl}}^{\text {core }}+F_{\mathrm{cl}}^{\text {corona }}$.

We assume normal distributions for proper motions of field and cluster stars. Also, the spatial distribution of cluster members is normal distribution, whereas field stars are uniformly distributed over the area. The parameters of the distribution functions were derived in a four-dimension space of proper motions $\left(\mu_{X}, \mu_{Y}\right)$ and plate coordinates
$(X, Y)$ by applying the maximum likelihood technique. To increase the stability of the solution, we only considered 656 stars with $V<16.0^{\mathrm{m}}$ and proper motion less than $50 \mathrm{mas} / \mathrm{y}$. The results of the solution are given in Table 3 . The cluster membership probabilities for the core and corona were computed as

$P(t)=\exp \left[-\frac{0.5}{K} \sum_{i=1}^{K}\left(t_{i}-\overline{t_{i}} / \sigma_{t_{i}}\right)^{2}\right] 100 \%$

where $t_{i}$ denote proper motions $\mu_{X}, \mu_{Y}$ or coordinates $X$, $Y(K=4) ; \overline{t_{i}}$ and $\sigma_{t_{i}}$ are the corresponding mean values and dispersions. The membership probabilities based on proper motions alone $(K=2)$ are also given in our 


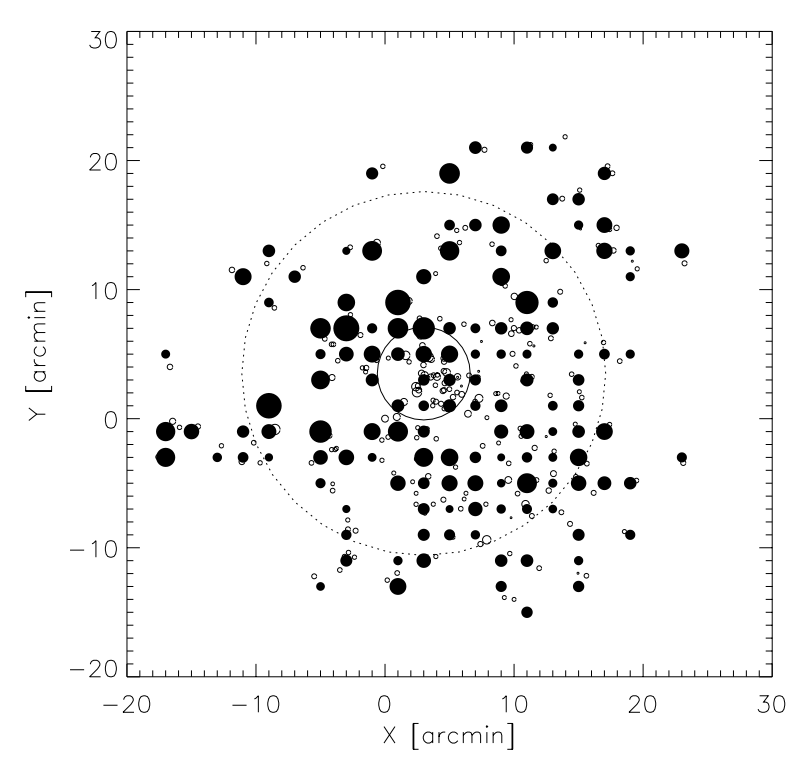

Fig. 6. Reddening map of the NGC 6611 region. The size of the filled circles is proportional to the color excess value averaged over $2^{\prime} \times 2^{\prime}$ cells. Stars from our catalogue brighter than $11.0^{\mathrm{m}}$ are plotted, too (open circles, with sizes proportional to stellar

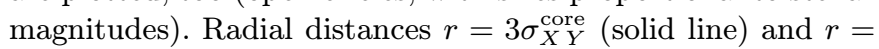
$3 \sigma_{X Y}^{\text {corona }}($ dashed line) are also indicated

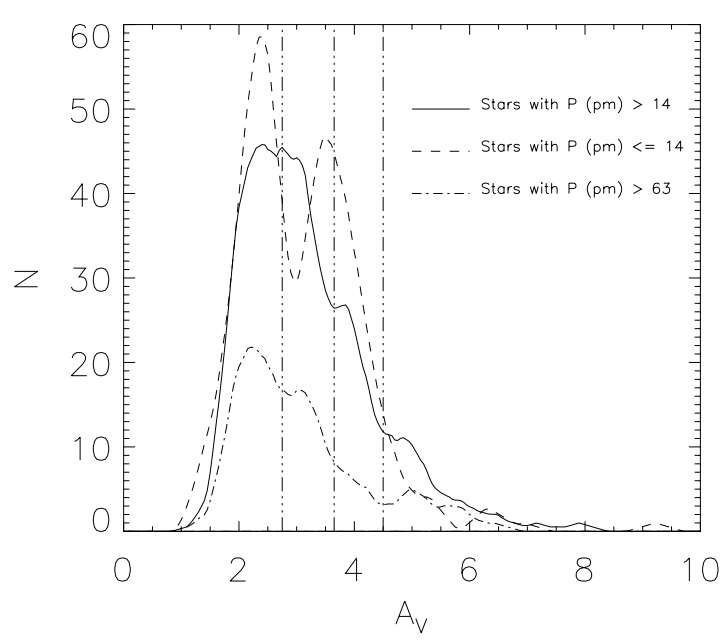

Fig. 7. Distribution of 467 catalogue stars with different cluster membership probabilities over $A_{V}$. Dotted-dashed line: stars with proper motion membership probability $P(\mathrm{pm})>63 \%$; solid line: stars with $P(\mathrm{pm})>14 \%$; dashed line: stars with $P(\mathrm{pm}) \leq 14 \%$. Four absorption groups showed in Fig. 8 are separated provisionally by dotted-dashed vertical lines

catalogue. The stars with membership probabilities higher than $61 \%, 14 \%, 1 \%$ are located at distances less than $1 \sigma$, $2 \sigma, 3 \sigma$ from the maximum of the distribution function and were considered as high probable, probable or low probable members, respectively.
Absolute proper motions of NGC 6611 along the $Y$ axis (direction of the Galactic rotation) and $Z$ axis (direction to the Galactic North Pole) are $+2.49 \pm 0.11$ and $-0.25 \pm$ $0.10 \mathrm{mas} /$ year, respectively. This corresponds to spatial velocity components of $V_{Y}=+24.8 \pm 1.1$ and $V_{Z}=-2.5 \pm$ $1.0 \mathrm{~km} \mathrm{~s}^{-1}$.

\section{Absorption and the color-magnitude diagram}

NGC 6611 is the extremely young open cluster associated with the nebula M 16. High and variable reddening and an anomalous extinction law were observed in this region (e.g. Sagar \& Joshi 1979; Thé et al. 1990; Hillenbrand et al. 1993; De Winter et al. 1997). Therefore, the assumption of an average value of the color excess $E_{(B-V)}$ and $R_{V}=A_{V} / E_{(B-V)}$ for all cluster stars may lead to incorrect results and conclusions by a study of cluster properties.

\subsection{Spatial distribution of absorption}

In order to construct the de-reddened color-magnitude diagram for the cluster, we need to know the distributions of color excesses and $R_{V}$ over the cluster area. Since data on individual values of interstellar absorption for cluster stars are rather poor, a reddening map may present a useful tool for the cluster studies. The first map of color excesses in NGC 6611 was constructed by Sagar \& Joshi (1979) on the basis of 50 stars in the inner cluster region $(20 \times 20$ sq. arcmin). Our catalogue supplemented by recently published extinction data of De Winter et al. (1997) gives a basis for the construction of a new reddening map in the NGC 6611 region.

To improve the statistics of individual $E_{(B-V)}$ and $R_{V}$ data, we applied the $Q$-method technique to multicolor CCD observations of Hillenbrand et al. (1993). Stars with $Q_{U B V}<-0.43^{\mathrm{m}}$ and $(B-V)<0.20^{\mathrm{m}}$ were taken into account and numerical parameters from Johnson (1966) and Hillenbrand et al. (1993) were used:

$Q_{U B V}=(U-B)-0.69(B-V)$,

$(B-V)_{0}=0.332 Q_{U B V}$

$(V-K)_{0}=1.05 Q_{U B V}$

and

$E_{(B-V)}=(B-V)-0.332 Q_{U B V}$

$R_{V}=1.1\left((V-K)-1.05 Q_{U B V}\right) /\left((B-V)-0.332 Q_{U B V}\right)$.

The following data sources (listed according to their priority) were included in the absorption study and reddening map construction:

- color excesses $E_{(B-V)}$ from our catalogue and $R_{V}$ taken from Thé et al. (1990) and De Winter et al. (1997); 


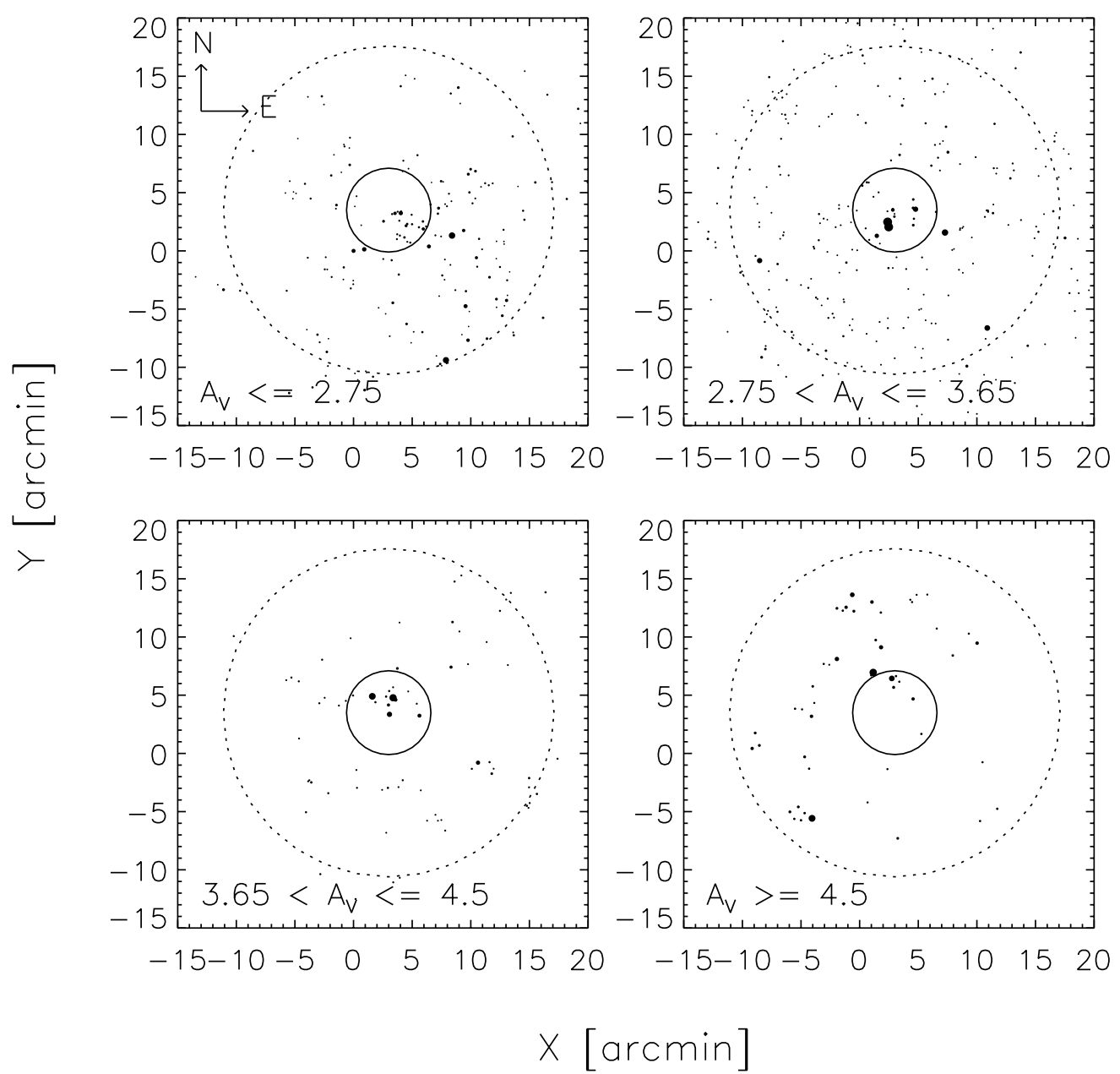

Fig. 8. Spatial distribution of 221 cluster members $(P(\mathrm{pm})>14 \%)$ belonging to the different absorption groups. Radial distances $r=3 \sigma_{X Y}^{\text {core }}$ (solid line) and $r=3 \sigma_{X Y}^{\text {corona }}$ (dashed line) are also indicated. Size of filled circles is proportional to $V$ magnitudes

- $E_{(B-V)}$ and $R_{V}$ calculated from Hillenbrand et al. (1993) CCD-photometry with the $Q$-method.

As a result, the sample for the absorption study includes 467 stars with color excesses (97 from this catalogue and 370 via the $Q$-method). For 174 of them, $R_{V}$ determinations are available (37 from De Winter et al. 1996 and 137 from the $Q$-method). According to the determined membership probabilities, this sample consists of 221 probable cluster members $(P(\mathrm{pm})>14 \%)$ and 246 field stars $(P(\mathrm{pm}) \leq 14 \%)$. For the construction of the reddening map, only cluster members were considered, whereas for the $R_{V}$-map all stars with known $R_{V}$ were used.

Color excesses and $R_{V}$ coefficients, averaged over small cells of $2 \times 2$ sq. arcmin are given in Table 4 (with $E_{(B-V)}$ as upper line and $R_{V}$ as lower line in each box). For illustration, we show in Fig. 6 the corresponding distribution of $E_{(B-V)}$ over the cluster area. The map covers a sky region of $42 \times 38$ sq. arcmin and, according to the cluster structure parameters given in Table 3, it includes both cluster core and corona.

The reddening map was used to study the distribution of absorption in the NGC 6611 area. For stars with known $E_{(B-V)}$ and $R_{V}$, the total absorption $A_{V}$ was calculated directly from the individual values. For cluster members with $R_{V}$ lacking, these values were taken, if available, from the reddening map. For remaining members an averaged value of $\overline{R_{V}}=3.75$ was adopted from Hillenbrand et al. (1993), whereas for field stars a value of 3.1 was assumed. The distribution of 467 stars of the sample as a function of $A_{V}$ is shown in Fig. 7. A kernel density estimation method with a triangular kernel was applied to compute a smoothed distribution:

$\mathrm{d} N / \mathrm{d} A_{V}=\phi\left(A_{V}\right)=\sum_{i} K\left(\frac{A_{V}}{h}\right)$

with

$K(x)= \begin{cases}1-|x|, & |x|<1.0 \\ 0, & |x| \geq 1.0\end{cases}$ 

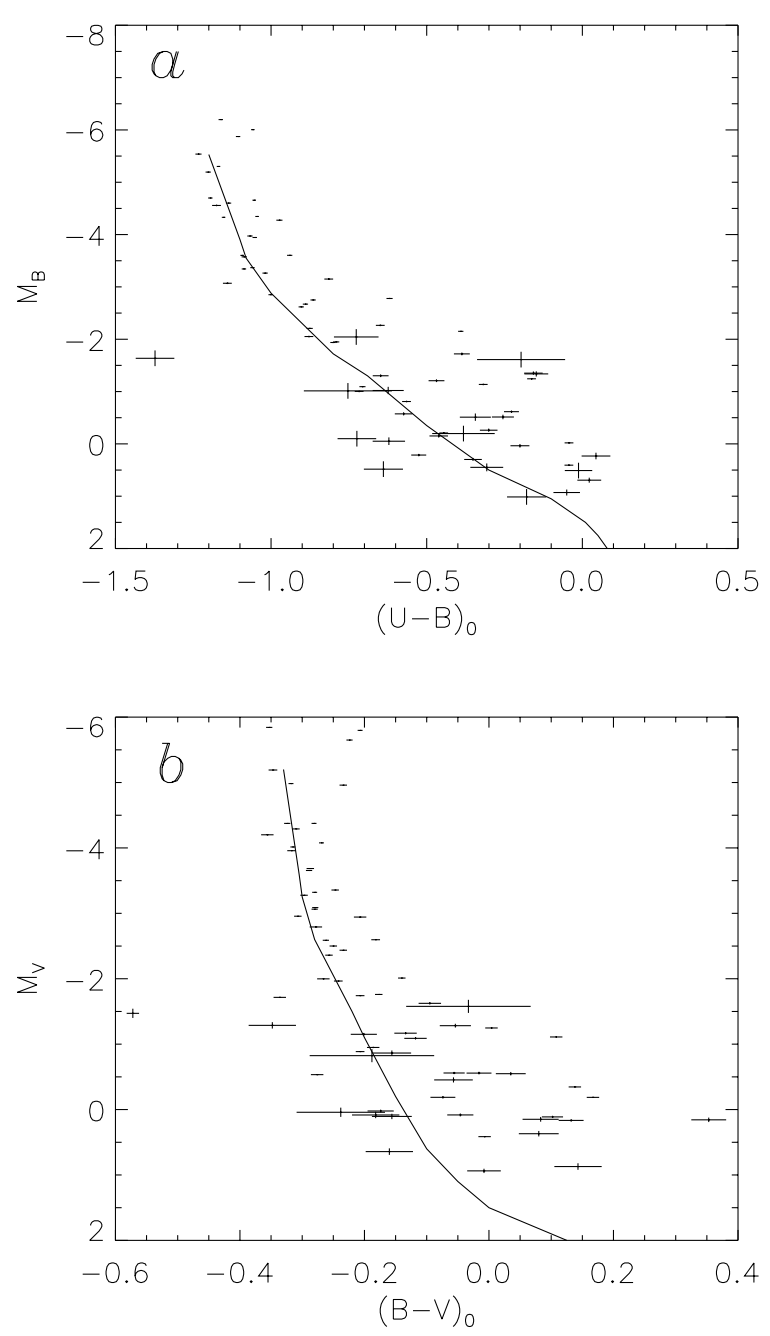

Fig. 9. Reddening free color-magnitude diagrams of NGC 6611 stars. Panel a) $M_{B}-(U-B)_{0}$, panel b) $M_{V}-(B-V)_{0}$. Stars with $P(\mathrm{pm}, x y)>63 \%$ are plotted only. Solid line is the ZAMS from Schmidt-Kaler (1982), horizontal and vertical bars indicate errors in colors and magnitudes, respectively

and $i$ running through the sample. According to preliminary tests on the smoothing parameter $h$ in an appropriate range of $[0.1,2.0]$, we chose $h=1.0$ as the best compromise to avoid statistical noise and to prevent an oversmoothing of the distribution.

According to Fig. 7, distributions of cluster members with proper motion probabilities $P(\mathrm{pm})>63 \%$ or $P(\mathrm{pm})>14 \%$ show a similar behaviour and differ significantly from the distribution of field stars. This fact may be interpretated as an independent evidence for the correctness of the kinimatic selection procedure.

In contrast to the distribution of the cluster members, the distribution of field stars shows two distinct components. We attributed a low-absorption peak at $A_{V} \approx 2.5^{\mathrm{m}}$ to the foreground field, while the second peak at $A_{V} \approx$ $3.7^{\mathrm{m}}$ includes background stars highly obscured by the cluster parent cloud. Unfortunately, we cannot make more concise quantitative conclusions due to strong selection effects influencing the sample of stars with available individual $E_{(B-V)}$ values.

The location of distribution features obtained for cluster candidates coincides well with the positions of the maxima of the $E_{(V-K)} / E_{(B-V)}$ distribution in Fig. 6 of Hillenbrand et al. (1993). Assuming an average of $E_{(B-V)}=0.79$, the peaks at $R_{V}=3.1$ and $R_{V}=3.75$ correspond to $A_{V}=2.5^{\mathrm{m}}$ and $A_{V}=3.0^{\mathrm{m}}$.

Considering the local minima in the cluster member distributions over $A_{V}$, we divided 221 cluster members $(P(\mathrm{pm})>14 \%)$ into four absorption groups indicated in Fig. 7 by vertical lines. The spatial distribution of these stars is shown in Fig. 8. This distribution confirms a patchy behavior of absorption over the cluster. The most obscured stars are observed within a strip located to the NW of the cluster core. The less obscured group $\left(3.65<A_{V} \leq 4.5\right)$ is randomly distributed within the core and corona whereas stars with $2.75<A_{V} \leq 3.65$ which could be considered as typical for this cluster unifomly fill the corona area. The stars absorbed least $\left(A_{V} \leq 2.75\right)$ mark a "transparency" window in the SE sector of the corona. Note that stars of other groups tend to avoid the window.

\subsection{Color-magnitude diagrams and distance}

The magnitudes taken from our catalogue were corrected for reddening by use of individual color excesses and the redenning map. In order to determine the distance modulus of the cluster, we constructed a color-magnitude diagram (CMD) for 72 probable cluster members with a membership probability higher than $63 \%$. Figure 9 shows both $M_{B}-(U-B)_{0}$ and $M_{V}-(B-V)_{0}$ CMDs constructed with

$$
\begin{aligned}
& M_{B}=M_{V}+(B-V)_{0}, \text { and } \\
& (U-B)_{0}=(U-B)-0.72 E_{(B-V)} .
\end{aligned}
$$

The ZAMS calibrations were taken from Schmidt-Kaler (1982). The distance modulus resulting from a fit of the ZAMS to the upper part of the CMDs $\left(M_{B}<-3^{\mathrm{m}}\right)$ was derived as $\left(m_{0}-M\right)=11.65^{\mathrm{m}} \pm 0.10^{\mathrm{m}}$ which is in good agreement with Hillenbrand et al. (1993) $\left(\left(m_{0}-M\right)=\right.$ $\left.11.5^{\mathrm{m}}\right)$. The corresponding distance is $2.14 \pm 0.10 \mathrm{kpc}$.

\section{Conclusions}

The main goal of this work was to gather and combine astrometric and photometric data obtained by different authors in the NGC 6611 region during the past 30 years. As a result of a careful analysis, a catalogue of about 
2000 stars was compiled which includes proper motions in the Hipparcos system and magnitudes, colours and extinction data in a common photoelectric system. Astrometric membership probabilities were computed and included in the catalogue, too. The data form an observational basis for further studies of this very young open cluster. The complete catalogue is available at cdsarc.u-strasbg.fr (CDS) via anonymous ftp.

Acknowledgements. We thank S. Röser for providing us with the stellar sample from AC/GSC catalogue. We thank the Karl-Schwarzschild-Observatorium Tautenburg for supplying and the MAMA team, particularly J. Guibert for measuring the plates. A. Belikov, N. Kharchenko and A. Piskunov thanks the Deutsche Forschungsgemeinschaft for financial support.

\section{References}

Bastian U., Röser S., 1993, PPM Star Catalogue 3, 4. Spectrum Academischer Verlag

Eichhorn H., 1974, Astronomy of star positions. Frederick Ungar Publishing Co., New York, p. 357

ESA, 1997, The Hipparcos and Tycho Catalogues, ESA SP-1200

Hillenbrand L.A., Massey P., Strom S.E., Merrill K.M., 1993, AJ 106, 1906
Hiltner W.A., Morgan W.W., 1969, AJ 74, 1152

Hoag A.A., Johnson H.L., Iriarte B., Mitchell R.I., et al., 1961, Publ. U.S. Naval Observ., 2nd Series 17, 347

Johnson H.L., 1966, Vgl. Ref. 823 S, 193

Kamp L.W., 1974, A\&AS 16, 1

Kharchenko N., Schilbach E., 1995, Astron. Nachr. 316, 91 (KS95)

Kharchenko N., Scholz R.-D., Lehmann I., 1997, A\&AS 121, 439

Morgan W.W., Whitford A.E., Code A.D., 1953, ApJ 118, 318

Nicolet B., 1978, A\&AS 34, 1

Röser S., 1996, in: Ferraz-Mello S., Morando B. and Arlot J.-E. (eds.) An updated GSC as the astrometric reference for minor planet observations. Kluwer Dordrecht, p. 481

Sagar R., Joshi U.C., 1979, Astrophys. Sp. Sci. 66, 3. Dordrecht, Reidel Publ. Comp., p. 451

Schmidt-Kaler Th., 1982, Landolt-Berstein Numerical Data and Functional Relationships in Science and Technology, New Series, Group IV. Berlin-Heidelberg, New York: Springer-Verlag Press 2, p. 15

Thé P.S., de Winter D., Feinstein A., Westerlund B.E., 1990, A\&AS 82, 319

Tucholke H.-J., Geffert M., Thé P.S., 1986, A\&AS 66, 311

Van Schewick H., 1962, Veröff. Univ. Sternw. Bonn 62, 1

Walker M.F., 1961, ApJ 133, 438

de Winter D., Koulis C., Thé P.S., van den Ancker M.E., Pérez M.R., Bibo E.A., 1997, A\&AS 121, 223 